EPJ Web of Conferences 40, 13005 (2013)

DOI: $10.1051 /$ epjconf/20134013005

(C) Owned by the authors, published by EDP Sciences, 2013

\title{
Modeling and experimental analysis of magnetostriction in high strength steels
}

\author{
H. ElBidweihy ${ }^{1}$, C. D. Burgy ${ }^{1,2}$, E. Della Torre ${ }^{1}$, and M. Wun-Fogle ${ }^{2}$ \\ ${ }^{1}$ Department of Electrical and Computer Engineering, The George Washington University, Washington, DC 20052, USA \\ ${ }^{2}$ Naval Surface Warfare Center, Carderock Division, West Bethesda, MD 20817, USA
}

\begin{abstract}
Previous studies on the magnetostriction in high strength steels have ignored the internal anisotropies due to previous material handling. Cold-rolling an iron alloy will stretch and distort the magnetic domains in the direction of rolling. These altered domain shapes impact the magnetic characteristics of the alloy; adding an additional preferred direction of magnetization to the easy or hard axes within the crystalline structure. This paper presents data taken on rods of a high strength steel that have been machined parallel to the rolling direction; as well as simulated results using a Preisach-type magnetostriction model. The model, whose formulation is based on the DOK magnetization-based model, aims specifically to simulate the Villari reversal phenomenon observed in the magnetostriction measurements of high strength steels and some Terfenol-D alloys.
\end{abstract}

\section{Introduction}

Magnetostriction is a mechanical strain caused by an applied magnetic field. High strength steels have magnetostrictions of $10-30 \times 10^{-6}$, which is one-half that of $\mathrm{Ni}$ for example. However, there are numerous situations in which the magnetostrictive properties of these steels are important; e.g. in torque sensing in shafts requiring high strength to weight ratios [1].

Steel shows inherently different material characteristics than giant magnetostrictive materials like Terfenol-D. A striking difference is shown in the "Villari reversal" seen in some high strength steels. This effect is characterized by the magnetostriction reaching a maximum value and then decreasing with larger applied fields instead of reaching a saturation value. Figure 1 illustrates an example of a Villari reversal, shown by the magnetostriction versus applied magnetic field for one of the parallel samples.

While previous work [1] took measurements principally in a single direction, we have taken into account directional anisotropies. Cold-rolling an iron alloy stretches and distorts the magnetic domains in the direction of rolling [2]. These altered domain shapes impact the magnetic characteristics of the alloy; adding an additional preferred direction of magnetization to the easy or hard axes within the crystalline structure. Some previous measurements have mentioned directional anisotropies within rolled steels but did not characterize the differences [3]. Our goal is to incorporate anisotropic stress-induced differences into a Preisach model. In a previous paper we have reported detailed measurement results for the magnetic properties of high strength steels oriented parallel, perpendicular and $45^{\circ}$ to the rolling direction [4].

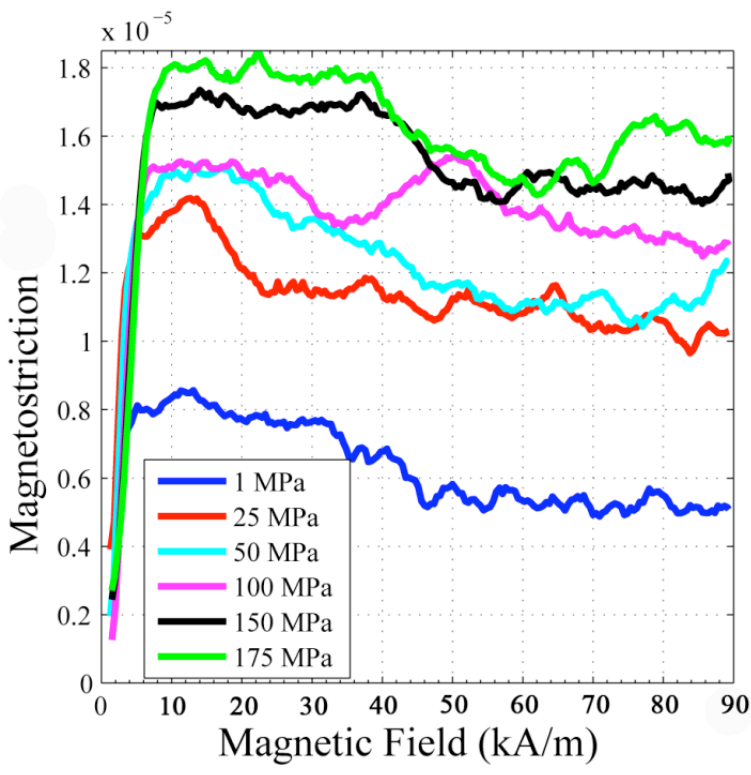

Fig. 1. Villari reversal in magnetostriction measurements of high strength steel under different compressive stress values.

This is an Open Access article distributed under the terms of the Creative Commons Attribution License 2.0, which permits unrestricted use, distribution, and reproduction in any medium, provided the original work is properly cited. 
In this paper we report comparisons of our previous magnetostriction measurements with predictions from a new Preisach-type model we recently developed [5]. The model simulates magnetostriction only, but its parameters are identified by the experimental measurements of: the magnetization, the magnetic susceptibility, and the magnetostrictive susceptibility. The measurements were conducted under compressive stresses between -1 and $175 \mathrm{MPa}$.

\section{Experiment}

Solid cylinders with their longitudinal axis oriented parallel, perpendicular and $45^{\circ}$ with respect to the rolling direction were machined from each of three locations on the original rolled plate of high strength steel, a total of nine samples. All of the samples were taken from the same sheet of rolled steel, and compositional differences between locations are assumed to be negligible. Consideration was given to the process of machining the samples from the rolled sheet, as the effect of adding additional stress to steel samples has been well documented [6]. In-depth details of this experiment and the sample compositions are shown in our previous paper [4].

Each cylinder was $\sim 5.71 \mathrm{~mm}$ in diameter and $\sim 55.8 \mathrm{~mm}$ in length. An MTS-858 hydraulic load frame compressed each sample under predetermined stresses while a longitudinal applied field $H_{\mathrm{L}}$ was varied. $H_{L}$ was actively controlled using a Hall probe and a PI controller to reduce the effect of the stress and field dependence of the magnetostrictive sample. Strain was measured by two MicroMeasurements WK-06-500GB-350 strain gages mounted on opposite sides of the rod with AE-15 resin. These strain gauges measured the mechanical load at the center of the sample. Other details of the experimental setup are given in [4]. The testing setup is shown in Fig. 2 .

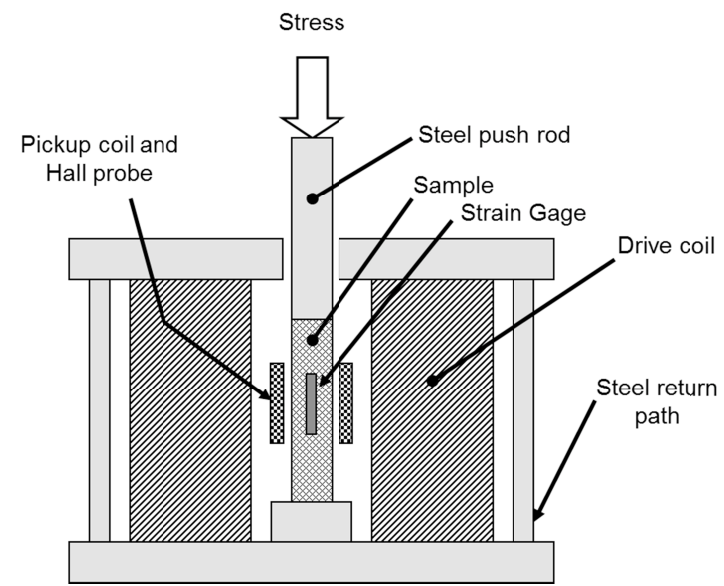

Fig. 2. Simplified experimental setup schematic. The closed-flux path provided by the load frame negates the demagnetization factor.

The experimental procedure started with a decreasing AC-field demagnetization of each sample in the longitudinal direction. The demagnetization was completed at $-1 \mathrm{MPa}$, which we have taken as zero applied stress due to the need for minor compression to hold the sample in place. The demagnetization loop consisted of 15.25 linearly decreasing sine cycles. The maximum $H_{\mathrm{L}}$ field was $149.6 \mathrm{kA} / \mathrm{m}$ and the frequency was $\sim 0.36 \mathrm{~Hz}$.

For the major loop measurements with fixed stress, once demagnetized, the sample was placed under the desired load of the measurement, and the strain gauges zeroed. Each cylinder was tested for 8 different fixed stresses between -1 and $-175 \mathrm{MPa}$. Then $H_{\mathrm{L}}$ was cycled over \pm $90 \mathrm{kA} / \mathrm{m}$. Each measurement set was preceded by this same demagnetization and compression cycle. Each of the measurements listed above were repeated for all nine cylinders. For the purposes of this paper, we have focused on the measurements and modeling of the parallel cylinders.

\section{Model Formulation}

We have developed a model for the hysteretic magnetostriction of high strength steels compressed under uniaxial stresses to specifically simulate the drop in the axial magnetostriction at higher magnetic fields [5]. This phenomenon is known in the literature as "Villari reversal" and can be described as follows: As the longitudinal magnetic field is increased under a compressive load, the axial magnetostriction in the direction of the applied field first increases to a maximum value and then drops gradually and eventually saturates.

This Preisach-type model approximates the magnetostriction's steep rise by a regulated irreversible magnetization process (first component) and the counteracting restoring torques by a reversible singlevalued magnetization process (second component). Two factors (A and B) are introduced whose sum is less than or equal to one. Factor $\mathrm{A}$ is a stress-dependent factor in which the stress magnitude affects the portion of magnetic domains initially lying in the perpendicular plane to the stress direction. Factor B is a balancing crystallographic-anisotropy factor which is determined by the restoring torques or the elastic binding forces which try to keep the magnetic domains oriented in a particular way.

The normalized strain $(\lambda)$ is given by:

$\lambda=\mathrm{A}(\sigma) * f(v \mathrm{H})+\mathrm{B}(\theta) * f(v \mathrm{H})$

where $\mathrm{H}$ is the longitudinal applied magnetic field, $\mathrm{A}$ is the stress-dependent factor which is a function of the compressive stress $(\sigma), \quad \mathrm{B}$ is the balancing crystallographic-anisotropy factor which is a function of the angle $(\theta)$ between the preferred orientation of the sample (the strain axis) and the magnetic field direction, $f$ is a monotonically increasing single-valued function, and $v$ is the magneto-elastic coefficient of the sample which is defined as the ratio of magnetostrictive susceptibility $\left(\mathrm{X}_{\lambda}\right)$ to magnetic susceptibility $\left(\mathrm{X}_{\mathrm{M}}\right)[7,8]$. 
The Preisach distribution of the model is incorporated in the A factor which is a function of the irreversible magnetization which in turn is calculated through the well-known Preisach function. For a DC-demagnetized single-quadrant material with a Gaussian Preisach function, the irreversible component of magnetization according to the DOK model is given by:

$\mathrm{MI}=\mathrm{S} * \mathrm{Ms} * \operatorname{erf}\left(\left(\mathrm{H}-\mathrm{H}_{\mathrm{rem}}\right) / \alpha\right)$

where $\mathrm{S}$ is the squareness, $\mathrm{Ms}_{\mathrm{s}}$ is the saturation magnetization, $\mathrm{Hrem}_{\mathrm{r}}$ is the remanent coercivity and $\alpha$ is the standard deviation of the switching field. For detailed explanation of the model's parameters and the mathematical expressions, refer to [8].

In order to evaluate the validity of the proposed model, a comparison between the measurements and the model's output is presented. The unknown parameters of the model were selected manually, yet selectively, to model the measurements. A detailed identification process will be the subject of a separate paper.

\section{Experimental Analysis \& Comparisons}

Figure 3 shows $v$ as a function of increasing magnetic field for different applied compressive stresses. For stress magnitudes lower than $125 \mathrm{MPa}, v$ decreases as the stress increases within the magnetostriction's steep rise region. Beyond this region (where the magnetostriction starts saturating), $v$ behaves the same regardless of the stress magnitude. For stress magnitudes higher than $125 \mathrm{MPa}$, $v$ is invariant with respect to stress magnitude. This observation is taken as a guide for choosing the value of $v$.

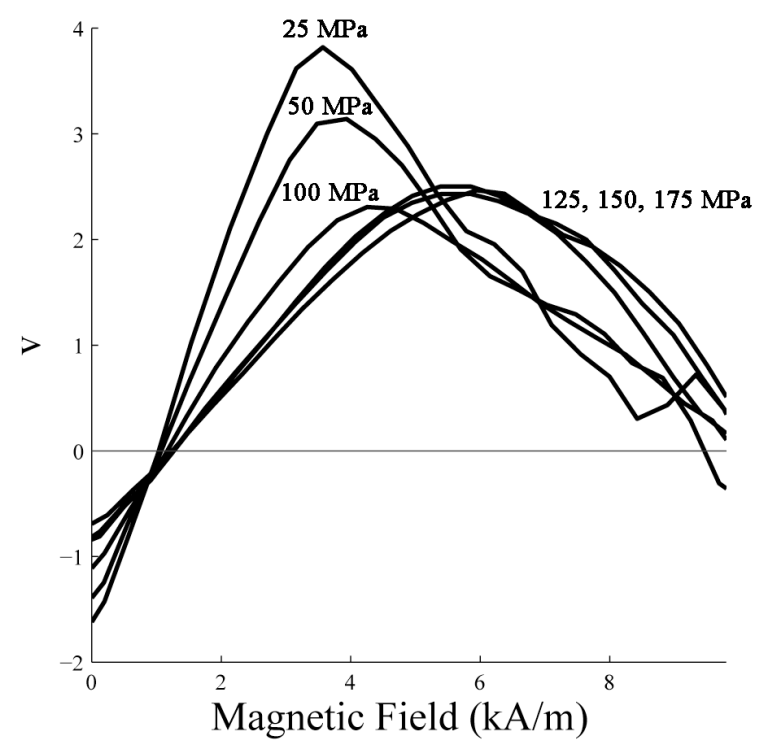

Fig. 3. The magnetoelastic coefficient $(v)$ as a function of increasing magnetic field $(\mathrm{H})$

Figure 4 shows the magnetostriction measurements as a function of the increasing magnetic field for different compressive stresses in the region of minimum magnetostriction. While the magnetostriction value where the dip occurs does not change consistently with the stress magnitude, the broadness of the flat region does. The width of the flat region increases with the stress magnitude. We also observed that the aforementioned width is strongly correlated to the peak of the magnetic susceptibility. This observation shall be indicative of the shift parameter which will be introduced to the model later on to simulate the hysteresis phenomenon. Again, beyond $125 \mathrm{MPa}$ the measurements are almost insensitive to the stress magnitude.

Figures 5-8 show comparisons between the measurements and the model results for three compressive stress magnitudes. The model shows qualitatively good results.

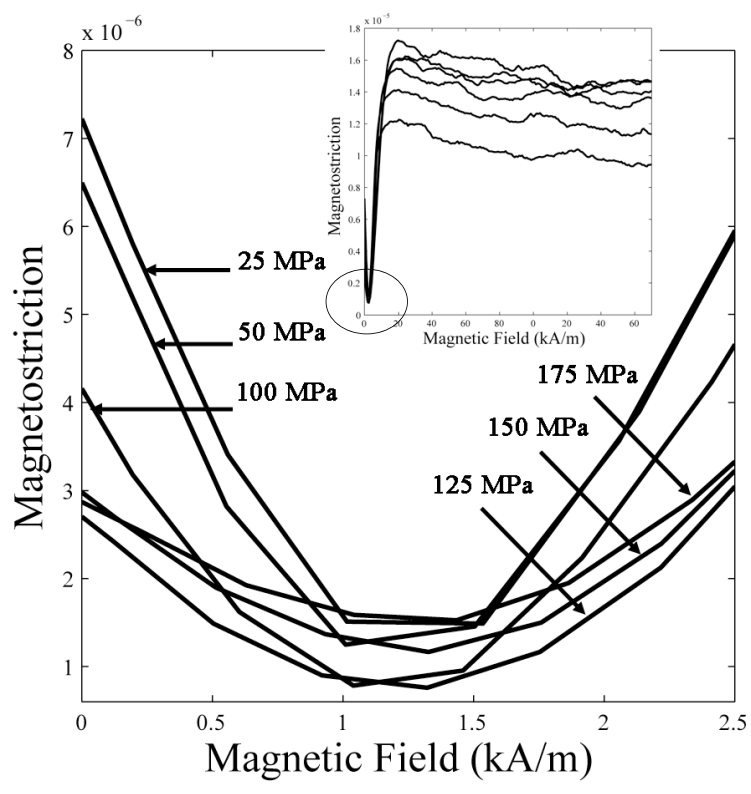

Fig. 4. Magnetostriction measurements as a function of increasing magnetic field $(\mathrm{H})$ at the region of minimum magnetostriction. Inset: Magnetostriction measurements with the region of interest circled 


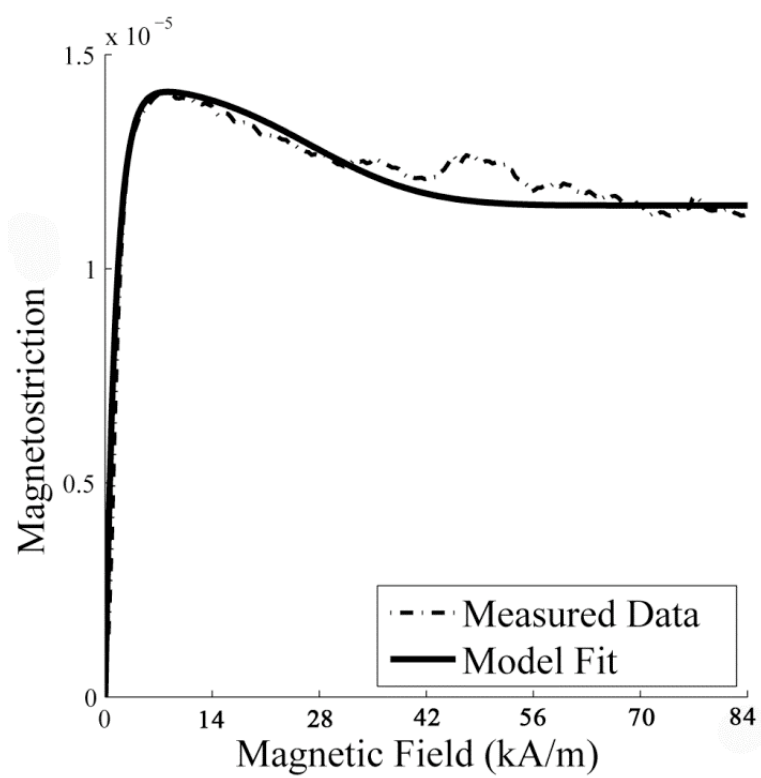

Fig. 5. Comparison between the magnetostriction measurements as a function of increasing magnetic field and the model results under a $50 \mathrm{MPa}$ compressive stress

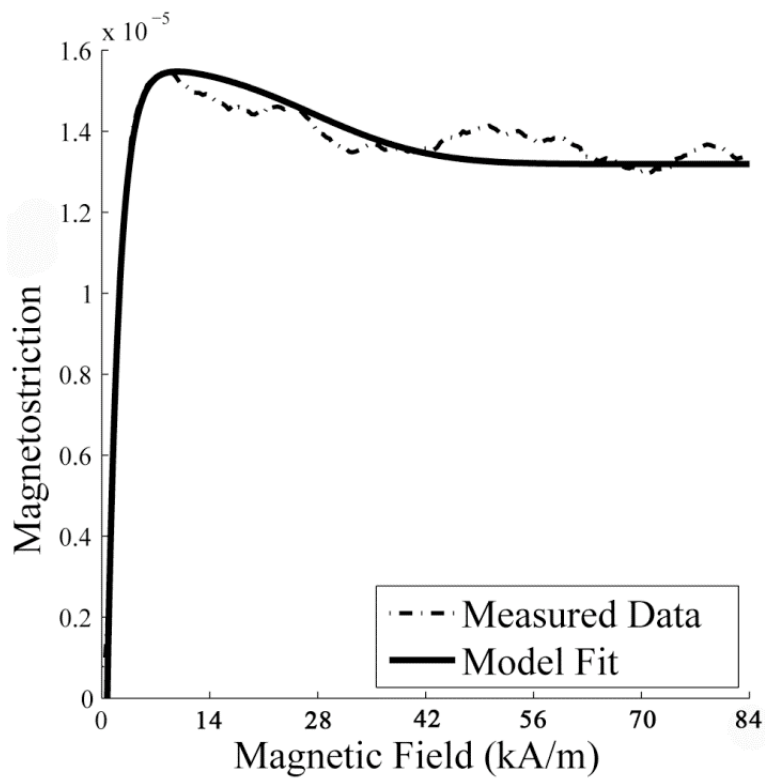

Fig. 6. Comparison between the magnetostriction measurements as a function of increasing magnetic field and the model results under a $100 \mathrm{MPa}$ compressive stress

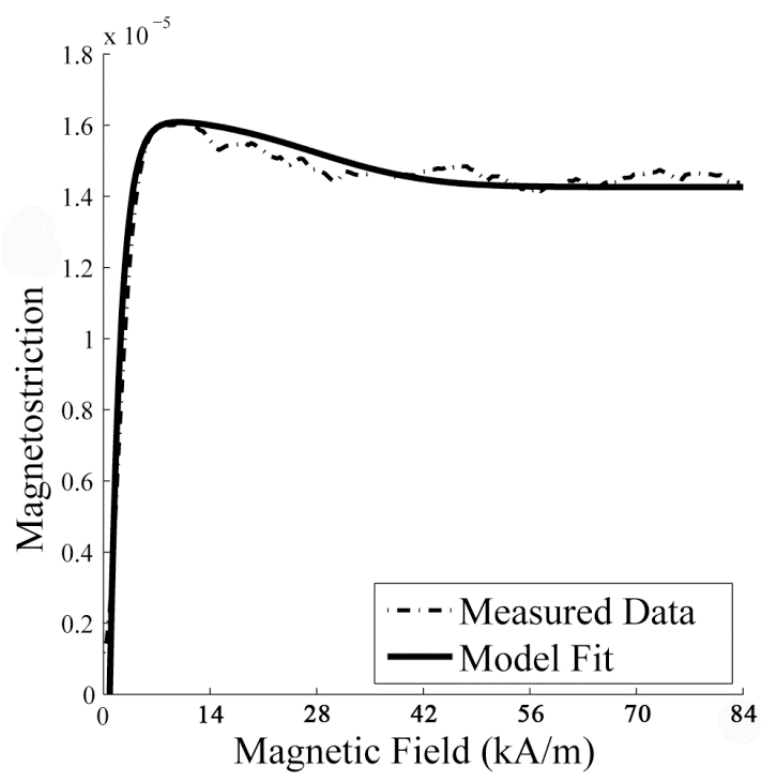

Fig. 7. Comparison between the magnetostriction measurements as a function of increasing magnetic field and the model results under a $150 \mathrm{MPa}$ compressive stress

\section{Conclusions}

Our previous measurements show interesting differences between the cylinders depending on their orientation with respect to the rolling direction. However, these differences diminish beyond a critical compressive stress magnitude, in our case this value is $125 \mathrm{MPa}$.

A Preisach-type, two-component model is presented to simulate the Villari reversal phenomenon observed in the magnetostriction measurements of high-strength steels under uniaxial compressive stress. The comparisons presented show that the model is able to simulate the Villari reversal observed in the measurements.

Future work entails investigating the hysteretic properties of the model as well as exploring the inverse magnetostriction phenomenon.

\section{References}

1. M. Wun-Fogle, J. B. Restorff, J. M. Cuseo, I.J Garshelis and S. Bitar, IEEE Trans. Magn., 45, 41124115 (2010)

2. R. M. Bozorth, Ferromagnetism (Wiley-VCH, 1993)

3. R. Grossinger, F. Keplinger, N. Mehmood, J. H. Espina-Hernandez, J. Araujo, C. Eisenmenger, K. Poppenberger, J. M. Hallen, IEEE Trans. Magn., 44, 3277-3280 (2008)

4. C. D. Burgy, E. Della Torre, M. Wun-Fogle, J. B. Restorff, IEEE Trans. Magn., 48, 3088-3091 (2012)

5. H. ElBidweihy, E. Della Torre, Y. Jin, L. H. Bennett, M. Ghahremani, IEEE Trans. Magn., 48, 3360-3362 (2012)

6. P. K. Klimczyk, P. Anderson, A. Moses, M. Davies, IEEE Trans. Magn., 48, 1417-1420 (2012)

7. E. Della Torre, A. Reimers, IEEE Trans. Magn., 33, 3967-3969 (1997)

8. E. Della Torre, Magnetic Hysteresis (Wiley-IEEE Press, 1999) 